\title{
Towards a seamless experimental protocol for human arm impedance estimation in an interactive dynamic task
}

\author{
Vincent Fortineau $^{1,2}$, Maria Makarov ${ }^{1}$, Pedro Rodriguez-Ayerbe ${ }^{1}$, Isabelle A. Siegler ${ }^{2}$
}

\begin{abstract}
The estimation of the human endpoint impedance interacting with a physical environment provides modelling insights both for the field of human movement science and for the design of innovative controllers for collaborative robotics based on physical human-robot interaction. Most of the existing human impedance estimation methods described in the literature rely on controlled environments closer to a laboratory than to an industrial setting. In this paper, a force perturbation method is proposed without any specific requirements on neither the force nor the position trajectories and without additional sensors placed on the human. The method is illustrated through an experimental study on a benchmark interaction task. The task was selected to be sufficiently variable for preventing the use of average trajectories and the perturbations used for the estimations are sufficiently low amplitude and short to not deteriorate the human performance in the task. The obtained impedance model parameters are in adequacy with the ones found in the literature, suggesting the validity of the proposed approach. The low constraints on the experimental settings make it applicable even out of the lab, for example, in humanrobot collaboration in manufacturing environments, to adjust the robot behaviour to changes in the state of the operator (fatigue, stress) or to adjust to a variety of human operators with different interaction strategies.
\end{abstract}

\section{INTRODUCTION}

Humans are capable of tailoring their limbs' dynamic properties [1] to improve the performance during the exploration of a new task [2] and achieve stable behaviour with a wide range of environments. Understanding and reproducing some properties of the humans' interactions during various tasks is thus relevant for the design of innovative robot control.

The mechanical impedance of human limbs has been studied for decades under the control prism [3] and is still an active research topic [4], [5]. The notion of impedance relates kinematics to forces, allowing the modelling of human movements during physical interactions. Elementary models, such as mass ( $M$ or $I$ for inertia) - spring $(K)$ - damper ( $B$, hence the acronym $K B M$ or $K B I$ ) have been shown to approach human behaviour [1].

The mechanical impedance cannot be directly measured and requires perturbing the limbs for estimations. Thus, the environment often has to be very well mastered to identify the impedance parameters. Two configurations can be easily distinguished: on one hand, static or isometric tasks [4], [6], where the unperturbed pose is well known and on the other

\footnotetext{
1 Université Paris-Saclay, CNRS, CentraleSupélec, Laboratoire des signaux et systèmes, 91190, Gif-sur-Yvette, France vincent. fortineaudcentralesupelec.fr

${ }^{2}$ CIAMS, Université Paris-Saclay, 91405, Orsay, France, \& Université d'Orléans, 45067, Orléans, France
}

hand, dynamic tasks, where ongoing movements make the estimation of the unperturbed trajectory more complicated.

The choice of the perturbations nature, either force or position, is often dictated by kinematics knowledge. When the trajectory can be estimated online [7], position perturbations enable stiffness identification alone. The introduction of force perturbations requires prior information on neither the movement nor the force shape. In [8], hand impedance estimations were conducted among welders, which brightly allowed the knowledge of the unperturbed trajectories. In other situations where the trajectory could not be perfectly known beforehand [7], [9], [10], the movement's repeatability allowed imperfect but sufficient knowledge on the unperturbed trajectories.

Another family of methods proposed in the literature relies on measurements of muscle activity using electromyographic sensors. Impedance estimations based on such measurements are reported in an isometric task [11] or right after its completion [12]. Dynamic tasks make it more complicated to differentiate impedance from the task muscles activation.

In previous work at the intersection of robotics, control and human movement sciences [13], the authors studied the human central nervous system during cyclic movements in a simulated benchmark task. They were able to reproduce human-like performances using a Central Pattern Generator based control structure. An experimental test-bed was designed for impedance measurements using interactive robotics [14].

The present paper aims to complete previous work by adding an impedance model of the human arm during the task. Dynamic considerations should allow finer modelling of human behaviour during a cyclic task, notably by observing possible impedance variations within a cycle or across individuals with different strategies. Such an examination provides insights for the human-robot collaboration and teleoperation as well as for control strategies design for robot interacting with various physical environments.

This paper proposes to identify the human endpoint impedance through an interactive robot [14], in conditions where both the restitution force and position (the differential between unperturbed and perturbed) can neither be measured, nor estimated with sufficient precision from average trajectories only. The new optimisation-based estimation of the unperturbed force trajectory proposed in this paper significantly improves the impedance parameter estimation. Experimental validation shows that the identified parameters are consistent with previously documented values (Section V-B3). Thus, the proposed estimation method allows less constrained experimental conditions and can be more easily replicated 
outside a laboratory for impedance estimation in a dynamic task.

The experimental test-bed is outlined in Section II, with the experimental data sets conditions. The issue of force trajectory estimation raised in [14] is then addressed in Section III. The impedance identification methodology is described in Section IV. Finally, the results using this implementation are presented in Section V.

\section{EXPERIMENT}

The experiment is briefly recalled in this section. For further details, please confer to previous work, [13], [14] for the ball bouncing task and [14] for the interactive robot control and a first approach of impedance estimation based on spline virtual trajectories.

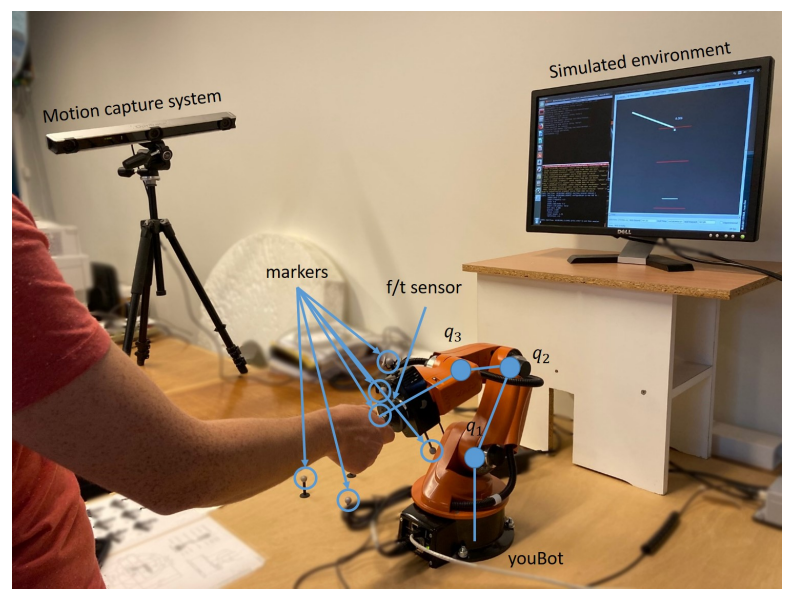

Fig. 1: Experimental set-up

\section{A. Interactive rhythmic task}

A user is manipulating an admittance controlled robot, manoeuvring its endpoint as a physical interface, to control a paddle simulated in a virtual environment (see Fig. 1). A 5-DOF KUKA ${ }^{\mathrm{TM}}$ youBot arm is used. The motion of the robot arm is restricted to the vertical plane using the control described in [14] and is performed using three actuated joints. To yield a rhythmic dynamic behaviour, the user has to move the paddle to bounce a virtual ball subject to gravity and moving along the vertical axis, to a target height simulated in the digital environment. When the ball hits the paddle, force feedback $f_{i}$ is provided by the robot to the human to simulate the impact of the ball on the paddle [15]. The motion generated can be compared to industrial rhythmic tasks such as stamping, punching or extended to planar movement such as sanding.

To allow impedance estimation during the task, pulse force perturbations are introduced using the robot, in both directions of the uni-dimensional task. Both timing and direction are stochastic. These perturbations are generated at the motor torque level and are consequently filtered by the robot dynamics before reaching the endpoint. In order to have a negligible influence on the task and approach a seamless methodology, the perturbations force effects have a duration under $60 \mathrm{~ms}$ and a rather low amplitude, below
$5 \mathrm{~N}$. Perturbations are pseudo-randomly introduced at three different phases of the cyclic behaviour. A cycle is defined here as the time frame between two consecutive ball impacts on the paddle. In the considered task, the mean cycle time is of $1.18 \mathrm{~s}$. According to the onset time in the cycle, the three perturbations categories are denoted as shown on Fig. 2 $c_{1}, c_{2}, c_{3}$.
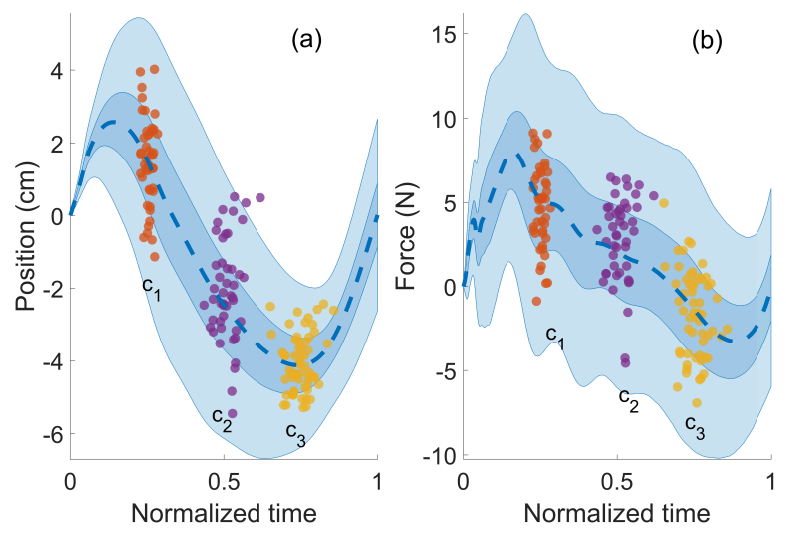

Fig. 2: Time normalised cycles of the data set $d_{3}$; perturbations are indicated with circles, the dotted lines represent the average cycles while the coloured areas represent 1 and 3 standard deviations from the average cycle. The time normalisation was done using interpolations on the average cycle duration.

\section{B. Data sets}

Both the endpoint position and interaction force between the human and robot arms are acquired with external sensors to obtain unbiased data. Interaction force is measured using an $\mathrm{ATI}^{\mathrm{TM}}$ mini45 force sensor also used in the robot admittance control and the endpoint position is measured using an Optitrack $^{\mathrm{TM}}$ V120:Trio motion capture system. For more information, please refer to [14].

A single user conducted a total of 28 physical interactions of approximately $5 \mathrm{~min}$ each with the experimental test-bed, without random perturbations. Half of those experiments (data set $d_{1}$ ) included force feedback for ball impacts (Section II-A). The other half $\left(d_{2}\right)$ included no force feedback on impacts.

A third data set, $d_{3}$, was collected by the same user. During a total of 7 manipulations of less than 2 min on average, 165 perturbed trajectories were recorded and classified according to the onset time of the force perturbation in the cycle (Section II-B). Eight of those perturbed trajectories could not be clearly classified because they occurred in unsteady cycles and were discarded. The distribution of onset time of the force perturbation is presented in Fig. 2. The three categories $c_{1}, c_{2}$ and $c_{3}$ are not exactly equally represented since their generation was pseudo-random, they have respectively 60 $(+28 /-32), 51(+25 /-26)$ and $46(+29 /-17)$ elements each. Each of these three categories contains positive and negative perturbations (numbers indicated previously in parentheses).

\section{VIRTUAL TRAJECTORIES ESTIMATION}

This section depicts the new methodology proposed to estimate unperturbed trajectories, previously called virtual trajectories (VT) in [14].

Most impedance estimation methods rely on the fact that deviations $\delta \boldsymbol{f}$ (force) and $\delta \boldsymbol{x}$ (position) from VTs (see 
Section IV for the impedance model relating motion to restitution force) can be introduced either by force or position perturbations. At least one is by essence unknown and needs to be reconstructed, while the other one can be perfectly mastered when the perturbation system allows it. In the literature, the averaged previous unperturbed trajectories were used in [9], with the assumption of sufficient reproducibility in the movements. A prediction algorithm with an unbiased error estimation, smaller than $1 \mathrm{~cm}$ after $200 \mathrm{~ms}$, was proposed in [7]. We could not reach better result than those with cubic splines [14] while replicating those methods, that were not designed for force trajectories with high variability, as is the case for the benchmark task considered in this study. The variability of position and force profiles is illustrated in Fig. 2.

\section{A. Preliminary remarks for signal analysis}

The estimation of the position and force VTs is first validated using the unperturbed data set $d_{2}$ (Section II-B). The position VT was well approximated with cubic splines in [14]. Accordingly, this part focuses on force trajectories.

1) Temporal analysis: As seen from Fig. 3, the force signals shape varies significantly across cycles, even in the absence of perturbations. While the positions follow roughly a sinusoidal profile (Fig. 2a), forces (Fig. 2b) are affected by higher frequency oscillations inside the cycle. These oscillations should not be considered as perturbations, and their origin can be related to the human-robot physical interaction and the admittance control tuning (while the stability of this control was experimentally verified, strict passivity was not ensured). This situation is not exceptional, all the more if several human subjects operate the same robot and employ different interaction patterns. These intra-cycle oscillations can not be simply filtered out and should be taken into account for an efficient VT estimation.
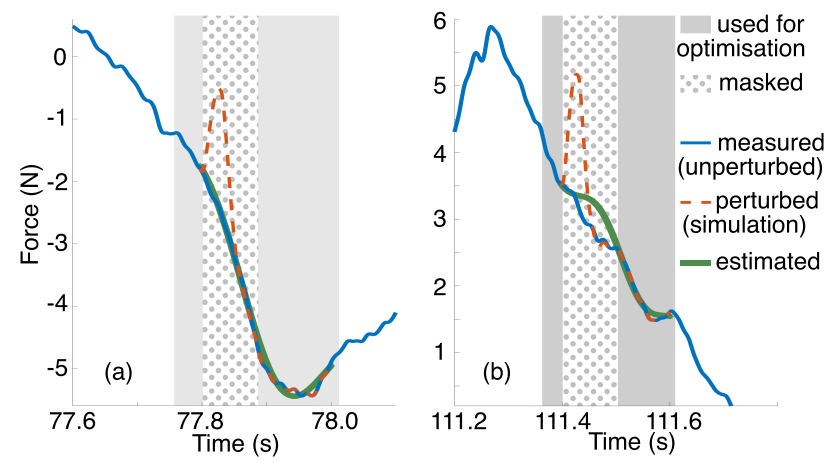

Fig. 3: Two examples of force trajectory estimations, with the measured unperturbed force (solid blue), the simulated perturbations (dashed orange) and estimated unperturbed force (green). Figure (a) depicts a successful estimation, while figure (b) shows a failed estimation that leads to an underestimated restitution force with oscillations.

2) Frequency analysis: In the Fourier transform of the data sets $d_{1}$ and $d_{2}$, the main frequency is observed around $0.9 \mathrm{~Hz}$, that is the frequency imposed by the ball bouncing task. While looking at the spectral analysis of both case scenario (with and without force feedback), the main differences occur after $5 \mathrm{~Hz}$ in both position and force signals. Those differences are more pronounced in the force spectral density, since the perturbations have more impacts on the force trajectory. Therefore, it can be deduced that most of the perturbed behaviour dynamics modify the trajectories, both in force and position, within a window of $200 \mathrm{~ms}$. It also is worth noticing that the force spectral density is larger, in a broader frequency band than the position, confirming that this signal is less filtered and richer in frequency content.

\section{B. Force virtual trajectory estimation}

The estimation of the virtual force using cubic spline interpolations [14] raised the issue of sensitivity to the landing point. Indeed, as advanced in Section III-A2, the force signal has higher power intensities, notably in higher frequencies. This makes the application of spline interpolation less reliable than for the position. In [10], the cyclic patterns of previous unperturbed data were fitted to a sinusoidal expression to predict the virtual position. A sum of sines is proposed to approach the virtual force in an offline resolution in a similar spirit. The general form is given in (1), with $n$ the number of sines; $A_{i}, f_{i}$ and $\phi_{i}$ the parameters of each sine. $a$ and $b$ the linear components, and $l$ a boolean. Hereinafter, this general form is adapted to the considered case through optimisations, and statistical analysis is performed.

$$
f_{z}(t)=l(a t+b)+\sum_{i=1}^{n} A_{i} \sin \left(2 \pi f_{i}+\phi_{i}\right)
$$

1) Optimisation: From (1), the tested subset of functions corresponds to $2 \leq n \leq 5$, which amounts to a total of 8 possible combinations with $l=0$ or 1 . To identify the parameters of these functions, an optimisation problem is defined to minimise the nonlinear quadratic error, $\min _{x}\left\|f\left(x=\left\{A_{i}, f_{i}, \phi_{i}, a, b\right\}\right)\right\|_{2}^{2}$, with a LevenbergMarquardt algorithm. Thereagainst [7], [10], the previous unperturbed cycle is not used to solve the VT, but only data within a cycle shortly before and after the perturbation; this is only possible since the estimation is proceeded offline (see Fig. 3). A chunk of the signal is selected with a masked window, corresponding to the perturbed force. The masked interval is chosen to be $100 \mathrm{~ms}$ long and will not be used to minimise the quadratic errors. $40 \mathrm{~ms}$ before the perturbation and $110 \mathrm{~ms}$ after the mask are used for the optimisation definition, as shown in Fig. 3.

This optimal problem is sensitive to its initialisation. Therefore, two options are put forward using $d_{2}$ : i) for each of the 14 sessions, the optimisation for the first cycle in the session is performed with 250 starting points and the parameters obtained from this optimisation are then used as initial values for the next cycles, ii) 50 starting points are used for every cycle.

\begin{tabular}{|c|c|c|c|c|c|}
\hline & $A_{i}(\mathrm{~N})$ & $f_{i}(\mathrm{~Hz})$ & $\phi_{i}\left(\mathrm{rad} \mathrm{s}^{-1}\right)$ & $a\left(\mathrm{~N} \mathrm{~s}^{-1}\right)$ & $b(\mathrm{~N})$ \\
\hline$b_{l}$ & 0 & 0 & $-p i / 2$ & -100 & -100 \\
\hline$x_{0}$ & 1 & $0.9(2 i-1)$ & 0 & $1 \mathrm{e}-3$ & $1 \mathrm{e}-3$ \\
\hline$b_{u}$ & 10 & 10 & $p i / 2$ & 100 & 100 \\
\hline
\end{tabular}

TABLE I: Lower $\left(b_{l}\right)$ and upper $\left(b_{u}\right)$ boundaries for the random initial starting points, and first manually fed initial condition $\left(x_{0}\right)$ 
2) Evaluation: In order to evaluate the proposed method, the estimated VTs need to be compared to a ground truth, unavailable in perturbed measurements. To that purpose, the sessions $d_{2}$ conducted without perturbations nor force feedback described in Section III-A are used. A total of 3144 evaluations are performed on the rhythmic force trajectories. If the optimised parameters are capable of approximating well the $100 \mathrm{~ms}$ masked $t_{\text {mask }}$ as well as the next $100 \mathrm{~ms}$ $t_{n e x t}$, then we consider that this methodology is capable of estimating a sufficient portion of the virtual force trajectory, for the impedance estimation, as explained in Section IV.

The comparison between the different models is then done using the Akaike criterion (AIC) to favour the accuracy of the model and penalise the number of parameters and, therefore, potential overfitting. The model with three sines $(n=3)$, no linear components $(l=0)$ and systematic multi-starts (option ii) reached the lowest AIC value and was therefore retained. This model has an average adjusted coefficient of determination $\left(R_{a d j}^{2}\right)$ of $91.0 \% \pm 10.0 \%$ on $t_{\text {mask }}+t_{\text {next }}$ interval and $95.1 \% \pm 5.3 \%$ on $t_{\text {mask }}$ interval after removing outliers outside five scaled median absolute deviations (sMAD). The distribution of the errors is Gaussianlike centred close to zero, and most of the errors occurred in the interval $t_{\text {mask }}$.

\section{IMPEDANCE IDENTIFICATION METHODOLOGY}

This section describes the methodology proposed for the identification of the impedance parameters.

This paper does not intend to differentiate the contribution of reflexes from the intrinsic properties of the human arm, like it was done in [16], but rather observe the arm's endpoint behaviour along the uni-dimensional task. A simple viscoelastic $2^{\text {nd }}$ order model of impedance is used (2), in a comparable manner as [8], with $\boldsymbol{M}, \boldsymbol{B}$ and $\boldsymbol{K} \in \mathbb{R}^{n_{c} \times n_{c}}$, respectively the matrices of mass, damping and stiffness in the $n_{c}$ Cartesians dimensions. For the studied uni-dimensional ball bouncing task, only the components along the vertical $z$ axis are considered, $n_{c}=1$.

$$
\delta \boldsymbol{f}=\boldsymbol{M} \delta \ddot{\boldsymbol{x}}+\boldsymbol{B} \delta \dot{\boldsymbol{x}}+\boldsymbol{K} \delta \boldsymbol{x}
$$

\section{A. Auto Regressive model}

1) Delay: Once the VTs have been computed from experimental data (see Section III), the differential estimated behaviour $\delta f_{z}$ and $\delta x_{z}$ can be exposed. Fig. 4 shows isolated perturbed behaviour of those trajectories. As expected from Section II-A, the main dynamics of the force perturbation happens in the first $60 \mathrm{~ms}$, with a maximum reached around $33 \mathrm{~ms}$. There is an apparent latency $\delta_{t}$ to notice in the positioning behaviour compared to the force differential. The figure does not allow an accurate evaluation of that delay; it can solely be bounded safely between 5 and $15 \mathrm{~ms}$. This delay is studied in Section V-A. A possible origin would be that the motion capture system, used for the position acquisition, has a sampling rate of $8.3 \mathrm{~ms}$, while position measurements were next interpolated at $1 \mathrm{kHz}$. A delay of about $8 \mathrm{~ms}$ can therefore be expected.
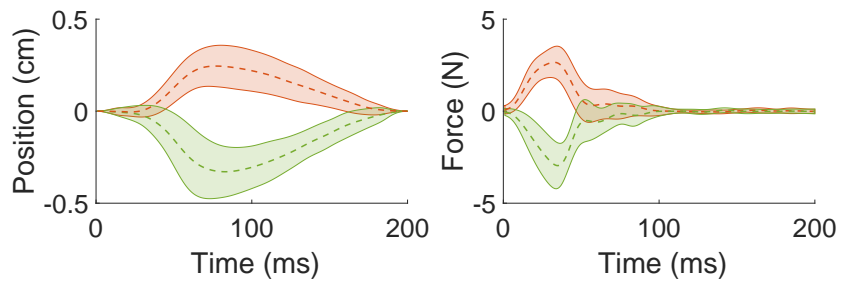

Fig. 4: Isolated typical effects of the perturbations on both the position and force. The average behaviours are shown with dotted lines, while the coloured areas represent the standard deviation. Positive (green) and negative (orange) perturbations are differentiated by their colour.

2) Identification model: The following discrete-time difference equation is considered for the identification of the second-order system (2) sampled with a zero-order hold and taking into account the previously mentioned pure delay:

$$
\left(1+a_{1} q^{-1}+a_{0} q^{-2}\right) \delta x_{z}\left[T_{k}\right]=\left(b_{1}+b_{0} q^{-1}\right) \delta f_{z}\left(T_{k}-\delta_{t}\right)+e\left(T_{k}\right)
$$

with $q$ the delay operator, $a_{i}$ and $b_{j}$ the model coefficients related to $K, B$ and $M, \delta_{t}$ a pure delay and $e$ white noise. The time window used for the identification is discussed in Section V-B1.

The eigenvalues of the discrete-time model (2) $z_{1,2}$ are related to the ones of the model (3) $z_{1,2}=\lambda_{1,2}$ via $e^{\lambda_{1,2} T}=\exp \left(0.5 T\left(-B \pm \sqrt{B^{2}+4 M K}\right) / M\right)=0.5\left(-a_{1} \pm\right.$ $\left.\sqrt{a_{1}^{2}-4 a_{0}}\right)$, with $T$ the sampling time.

The stiffness parameter of (2) corresponds to the static gain and thus from (3), $K=\left(1+a_{1}+a_{0}\right) /\left(b_{1}+b_{0}\right)$.

Identifying the impedance parameters $K, B$ and $M$ using this ARX model appears to be more numerically robust than with a straight temporal least square identification technique based on velocities and accelerations. Indeed, this way, the kinematic derivatives are not required, either using sensors or using numerical derivation.

\section{B. Model evaluation}

1) Simulation: A simulation was used to validate both the presented VT estimation and impedance parameters identification methods. In an over-simplistic spirit, the model for the arm movements (trajectory control) was assumed to be the same as the one for the impedance behaviour (disturbance rejection control). For the sake of veracity, empirical force data was injected into the arm model. Force perturbations of $5 \mathrm{~N}$ amplitude were generated with a second-order filter of $70 \mathrm{~ms}$ pseudo-period to resemble the experimental ones. Those perturbations were injected in an impedance model with known parameters and summed to the simulated arm movement. For simulated impedance behaviours given in Table II, conducted on 104 estimations, an average $R_{a d j}^{2}$ of $99 \% \pm 1 \%$ was reached to reconstruct the position with the identified parameters after removing outliers (5 sMAD). The stiffness was the parameter with the highest volatility, therefore to abide by the increase in abnormal data, the threshold was reduced from five to the usual three sMAD.

It can be noticed that with perfect knowledge of the VT, the parameters were estimated with relative errors of about $0.05 \%$, which means that the estimation errors mainly originate from the errors of VT estimation. 


\begin{tabular}{|c|c|c|c|c|c|c|}
\hline & $\hat{K}_{1}$ & $\hat{B}_{1}$ & $\hat{M}_{1}$ & $\hat{K}_{2}$ & $\hat{B}_{2}$ & $\hat{M}_{2}$ \\
\hline mean & $258 \mathrm{~N} \mathrm{~m}^{-1}$ & $12.5 \mathrm{~N} \mathrm{~s} \mathrm{~m}^{-1}$ & $523 \mathrm{~g}$ & $378 \mathrm{~N} \mathrm{~m}^{-1}$ & $15.9 \mathrm{~N} \mathrm{~s} \mathrm{~m}^{-1}$ & $487 \mathrm{~g}$ \\
\hline std & $116 \mathrm{~N} \mathrm{~m}^{-1}$ & $6.81 \mathrm{~N} \mathrm{~s} \mathrm{~m}^{-1}$ & $110 \mathrm{~g}$ & $166 \mathrm{~N} \mathrm{~m}^{-1}$ & $5.89 \mathrm{~N} \mathrm{~s} \mathrm{~m}^{-1}$ & $63 \mathrm{~g}$ \\
\hline$r e^{(2)}$ & $29 \%$ & $25 \%$ & $5 \%$ & $5.4 \%$ & $6.0 \%$ & $2.5 \%$ \\
\hline exact $^{(3)}$ & $200 \mathrm{~N} \mathrm{~m}^{-1}$ & $10.0 \mathrm{~N} \mathrm{~s} \mathrm{~m}^{-1}$ & $500 \mathrm{~g}$ & $400 \mathrm{~N} \mathrm{~m}^{-1}$ & $15.0 \mathrm{~N} \mathrm{~m}^{-1}$ & $500 \mathrm{~g}$ \\
\hline
\end{tabular}

TABLE II: Parameters estimation in simulation.

\section{Results}

This section presents the results obtained using the experimental protocol previously described in [14], with force feedback and controlled timing of force perturbations. The data set $d_{3}$, described in Section II-B, is used. Variance analysis (ANOVA) is conducted with a threshold fixed at $5 \%$ for significance levels. Both the degrees of freedom and the $\mathrm{F}$ value will be indicated in parenthesis $(\mathrm{DOF}, \mathrm{F})$ for the ANOVAs.

\section{A. Delay}

The delay $\delta_{t}$ unveiled in Section IV-A1 was determined by running several estimations with varying delay. To evaluate the quality of estimated parameters, the measured position was compared to its reconstruction using the identified parameters and the estimated input force. A one-way ANOVA showed a significant main effect of the delay on $R_{a d j}^{2}(10,14.75)$. The highest adjusted coefficient of determination reached a score of $95.1 \% \pm 5.5 \%$ for $12 \mathrm{~ms}$ ( 5 sMAD); this delay is therefore used for the impedance estimations. Although it is important to stress that a posthoc analysis (Tukey's HSD) revealed that no significant differences were observed between the $12 \mathrm{~ms}$ delay and delays comprised between $8 \mathrm{~ms}$ to $14 \mathrm{~ms}$.

\section{B. Impedance parameters}

1) Time window: As mentioned in [8], voluntary reactions need to be discarded because linear impedance models cannot correctly model them. Voluntary reactions can be observed as soon as $100 \mathrm{~ms}$ after a perturbation [17]. Yet, at a $1 \mathrm{kHz}$ rate, [8] found that using only $100 \mathrm{~ms}$ of data after the perturbation did not bring satisfying results. Therefore, they assumed that voluntary actions were not dominant in the first $200 \mathrm{~ms}$ and used that time window for the evaluation. To confirm that observation, impedance estimations were conducted with a data range varying from $50 \mathrm{~ms}$ to $200 \mathrm{~ms}$.

Fig. 5 shows the average estimated stiffness, damping and mass values as functions of the estimation time window length. Thoses average values no longer vary starting from approximately $150 \mathrm{~ms}$ estimation time windows. However, the standard deviation of each of the parameter seems to reach a steady-state before, for about $80 \mathrm{~ms}$, without significant reductions after that milestone. At the final stage, for $200 \mathrm{~ms}$, the stiffness still has a standard deviation that represents $44.8 \%$ of its average value, the damping $34.2 \%$ and the mass $42.9 \%$. This variability might be explained by the nondifferentiation of the different perturbation categories. This result tends to show that an identification on a time window of $150 \mathrm{~ms}$ at $1 \mathrm{kHz}$ might be enough. The paper will study both the identified parameters using $150 \mathrm{~ms}$ (subscript $i$ ) and $200 \mathrm{~ms}$ (subscript $\mathrm{ii}$ ) to carry this investigation.
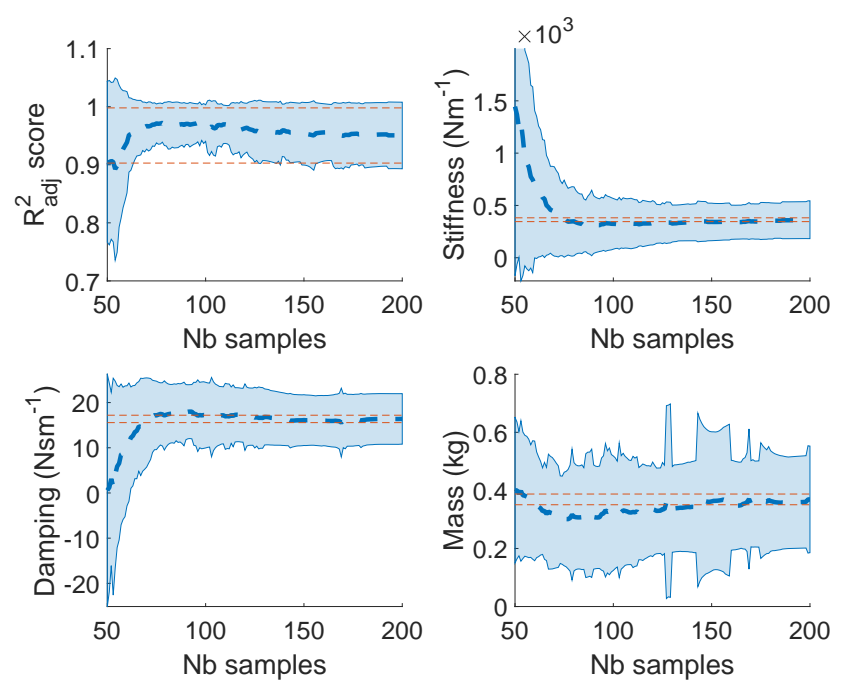

Fig. 5: Influence of the number of samples used for the ARX identification, the blue dotted line represents the average, and the light blue area represents one standard deviation from the average. The orange dotted lines show the $5 \%$ boundaries of the final values.

2) Outliers: The impedance estimations were separated into several groups according to the cyclic phase and the perturbation direction to isolate possible sources of variability. For both identifications $(i, i i)$, most of the outliers happened in a single class $c_{2}+$ (see Fig. 6). Among the 157 classified recordings, a total of 14 values were not considered $(8.9 \%)$. Similar results were found for the identification $i$, but with a total of 17 outliers, which represent $10.8 \%$ of the data.
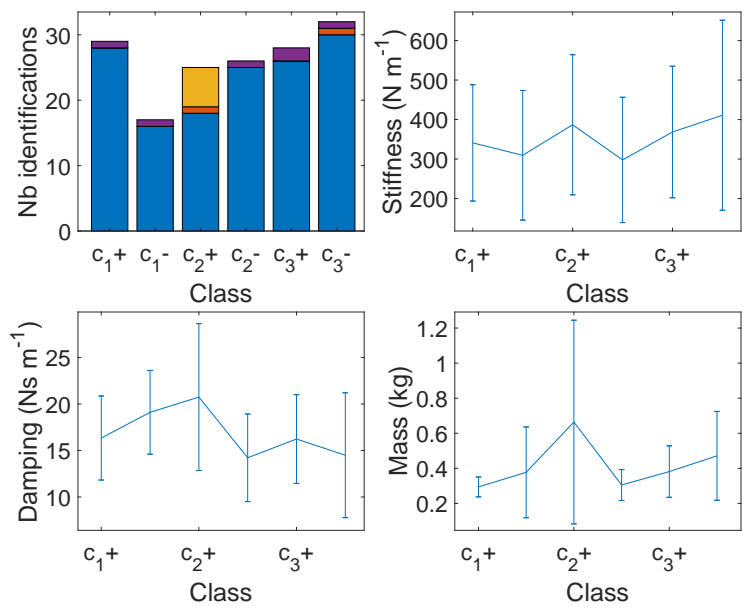

Fig. 6: The bar stacked chart shows in blue recordings kept for further analysis, in orange and purple respectively dismissed recordings with $R_{a d j}^{2}<50 \%$ (type 1) and stiffnesses outside 5 sMAD (type 2). The yellow indicates the outliers that belong to both to type 1 and 2 . The others charts indicate the average and standard deviation for all impedance parameters estimated using the valid recordings and $200 \mathrm{~ms}$ (ii).

3) Estimated parameters: In [1], [3], [6], [8], Cartesian stiffnesses were found within a magnitude ranging in $40 \mathrm{~N} \mathrm{~m}^{-1}$ to $700 \mathrm{~N} \mathrm{~m}^{-1}$, under static and moving configurations, with and without load for the human arm. Moreover [1], [6], [8] found damping parameters ranging between $8 \mathrm{~N} \mathrm{~s} \mathrm{~m}^{-1}$ to $44 \mathrm{~N} \mathrm{~s} \mathrm{~m}^{-1}$ and masses between $0.6 \mathrm{~kg}$ to $2.5 \mathrm{~kg}$. The results presented here (Table III) are within those ranges but for the estimated masses that are slightly below. 


\begin{tabular}{|c|c|c|c|c|c|c|c|c|}
\hline & $c_{1}^{+}$ & $c_{1}^{-}$ & $c_{2}^{+}$ & $c_{2}^{-}$ & $c_{3}^{+}$ & $c_{3}^{-}$ & + & - \\
\hline${ }_{i} K$ & $338 \pm 158$ & $319 \pm 151$ & $408 \pm 227$ & $294 \pm 173$ & $308 \pm 133$ & $397 \pm 221$ & $345 \pm 172$ & $343 \pm 193$ \\
\hline${ }_{i} B$ & $16.7 \pm 5.2$ & $18.5 \pm 4.2$ & $17.8 \pm 12.0$ & $13.7 \pm 4.4$ & $15.6 \pm 6.6$ & $13.2 \pm 5.4$ & $16.6 \pm 7.8$ & $14.6 \pm 5.2$ \\
\hline${ }_{i} M$ & $0.288 \pm 0.061$ & $0.369 \pm 0.235$ & $0.756 \pm 0.788$ & $0.293 \pm 0.071$ & $0.354 \pm 0.123$ & $0.484 \pm 0.393$ & $0.430 \pm 0.441$ & $0.391 \pm 0.291$ \\
\hline${ }_{i} R_{a d j}^{2}$ & $83.5 \pm 4.6$ & $88.7 \pm 3.9$ & $73.6 \pm 14.0$ & $93.0 \pm 2.9$ & $81.6 \pm 9.8$ & $82.6 \pm 6.9$ & $80.3 \pm 10.2$ & $87.6 \pm 6.9$ \\
\hline${ }_{i i} K$ & $340 \pm 147$ & $310 \pm 164$ & $387 \pm 177$ & $298 \pm 159$ & $368 \pm 167$ & $411 \pm 241$ & $362 \pm 160$ & $348 \pm 203$ \\
\hline${ }_{i i} B$ & $16.3 \pm 4.5$ & $19.1 \pm 4.5$ & $20.7 \pm 7.9$ & $14.2 \pm 4.7$ & $16.2 \pm 4.8$ & $14.5 \pm 6.7$ & $17.4 \pm 5.9$ & $15.4 \pm 5.9$ \\
\hline${ }_{i i} M$ & $0.294 \pm 0.057$ & $0.378 \pm 0.259$ & $0.664 \pm 0.581$ & $0.305 \pm 0.088$ & $0.381 \pm 0.147$ & $0.471 \pm 0.253$ & $0.418 \pm 0.334$ & $0.392 \pm 0.222$ \\
\hline${ }_{i i} R_{a d j}^{2}$ & $88.1 \pm 3.6$ & $91.6 \pm 3.0$ & $80.5 \pm 11.4$ & $94.0 \pm 7.5$ & $86.4 \pm 7.0$ & $84.7 \pm 6.8$ & $80.4 \pm 10.7$ & $85.6 \pm 10.4$ \\
\hline
\end{tabular}

TABLE III: Results: standard deviation are indicated in as confidence intervals.

4) Variance analysis: Considering the results from the identifications using $150 \mathrm{~ms}$, an analysis of variance showed a significant effect of the phase on the adjusted coefficients of determination $(5,15.12)$. However, a posthoc analysis revealed that differences between positive and negative perturbations were only significant within $c_{2}$. Only one significant difference could be observed between the classes $c_{1}$ and $c_{3}$, for the specific case of $c_{1}^{-}$and positive $c_{3}^{+}$. Taking into account the impedance parameters, neither the mean variations of stiffness nor damping observed during the different phases were significant $(5,1.59),(5,2.37)$.

Similar results were found for the identifications done with $200 \mathrm{~ms}$. The only noticeable discrepancy was about the damping values identified, which were found significantly different $(5,4.34)$, solely because of group $c_{2}^{+}$. Finally, comparing $i$ and $i i$ identifications, in first hand, if regrouping all classes, significant differences could only be found for $R_{a d j}^{2}(1,12.03)$ with an increase of $4 \%$ in average for the second identification and non-meaningful mean variations of the impedance parameters between $1 \%$ and $5 \%$. On the other hand, if comparing separated classes in both identification, (eg. ${ }_{i} c_{1}^{+}$with ${ }_{i i} c_{1}^{+}$), no significant differences were found.

\section{CONCLUSiONS}

The identification method proposed here with the experimental test-bed design described in [14], offers a lowconstraint methodology for the impedance identification in the absence of knowledge on both force and position VTs during a dynamic task. Estimated endpoint impedance parameters obtained with the proposed method are within the range found in the literature. However, the identification being sensitive to errors in the VTs as well as non-linear phenomena (voluntary movements, non-linear reflexes), the method still requires numerous estimations for reliable results. The results in this paper tend to show that using a time window of only $150 \mathrm{~ms}$ for the identification was sufficient to bring result non significantly different from those with a $200 \mathrm{~ms}$ time window.

No significant stiffness variations were observed in the three cyclic phases chosen. Most of the significant variations were concentrated in class $c_{2}^{+}$. Compared to previous work, using an ARX methodology improved the repeatability in the estimation of both the damping and mass. Using the new force trajectory estimation method drastically reduced the errors in VTs and improved the estimation of the stiffness, for perturbation below $5 \mathrm{~N}$ inducing typical movements deviations $\delta x$ less than $1 \mathrm{~cm}$. It made the perturbations hardly distinguishable from the task force feedback without disrupting the task.

Further experiments will be conducted to evaluate the inter and intra-individual variability of end-point impedance parameters in interactive tasks. Obtained parameter values can be used for the analysis and design of feedback control strategies in interactive robotics.

\section{REFERENCES}

[1] T. Tsuji and Y. Tanaka, "Bio-mimetic impedance control of robotic manipulator for dynamic contact tasks," Rob Auton Syst., vol. 56, pp. 306-316, Apr. 2008.

[2] E. Burdet, R. Osu, D. W. Franklin, T. E. Milner, and M. Kawato, "The central nervous system stabilizes unstable dynamics by learning optimal impedance," Nature, vol. 414, pp. 446-449, Nov. 2001.

[3] F. A. Mussa-Ivaldi, N. Hogan, and E. Bizzi, "Neural, mechanical, and geometric factors subserving arm posture in humans," J. Neurosci., vol. 5, pp. 2732-2743, Oct. 1985.

[4] C. Fang, A. Ajoudani, A. Bicchi, and N. G. Tsagarakis, "Online Model Based Estimation of Complete Joint Stiffness of Human Arm," IEEE Robot. Autom. Lett., vol. 3, pp. 84-91, Jan. 2018.

[5] G.-H. Phan, C. Hansen, P. Tommasino, et al., "Estimating Human Wrist Stiffness during a Tooling Task," Sensors, vol. 20, p. 3260, Jan. 2020. Number: 11 Publisher: Multidisciplinary Digital Publishing Institute.

[6] J. M. Dolan, M. B. Friedman, and M. L. Nagurka, "Dynamic and loaded impedance components in the maintenance of human arm posture," IEEE Trans. Syst. Man Cybern. Syst., vol. 23, pp. 698-709, May 1993.

[7] E. Burdet, R. Osu, D. W. Franklin, T. Yoshioka, T. E. Milner, and M. Kawato, "A method for measuring endpoint stiffness during multijoint arm movements," J. Biomech., vol. 33, pp. 1705-1709, Dec. 2000.

[8] M. S. Erden and A. Billard, "Hand Impedance Measurements During Interactive Manual Welding With a Robot," IEEE Trans. Robot., vol. 31, pp. 168-179, Feb. 2015.

[9] D. J. Bennett, J. M. Hollerbach, Y. Xu, and I. W. Hunter, "Time-varying stiffness of human elbow joint during cyclic voluntary movement," Exp Brain Res, vol. 88, pp. 433-442, Feb. 1992.

[10] M. O. Abe and N. Yamada, "Modulation of elbow joint stiffness in a vertical plane during cyclic movement at lower or higher frequencies than natural frequency," Exp Brain Res, vol. 153, pp. 394-399, Dec. 2003.

[11] C. Wang, L. Jiang, C. Guo, Q. Huang, B. Yang, and H. Liu, "sEMGbased estimation of human arm force using regression model," in 2017 IEEE Int. Conf. Rob. and Biomim. (ROBIO), pp. 1044-1049, Dec. 2017.

[12] C. Castellini, A. Arquer, and J. Artigas, "sEMG-based estimation of human stiffness: Towards impedance-controlled rehabilitation," in Proc. IEEE RAS EMBS Int. Conf. Biomed. Robot. Biomechatronics, pp. 604609, Aug. 2014. ISSN: 2155-1782.

[13] G. Avrin, I. A. Siegler, M. Makarov, and P. Rodriguez-Ayerbe, "The self-organization of ball bouncing," Biol Cybern, vol. 112, pp. 509-522, Dec. 2018.

[14] V. Fortineau, M. Makarov, P. Rodriguez-Ayerbe, and I. A. Siegler, "Interactive robotics for human impedance estimation in a rhythmic task," in 2020 IEEE 16th Int. Conf. on Autom. Science and Engineering (CASE), pp. 1043-1048, Aug. 2020. ISSN: 2161-8089.

[15] Y. Kawazoe, "Dynamics of the Ball-Racket Impact in Tennis : Contact Force, Contact Time, Coefficient of Restitution, and Deformation," Asian Conf. on Multibody Dyn., vol. 2002, pp. 286-293, 2002.

[16] D. L. Guarin and R. E. Kearney, "Unbiased Estimation of Human Joint Intrinsic Mechanical Properties During Movement," IEEE Trans. Neural Syst. Rehabilitation Eng., vol. 26, pp. 1975-1984, Oct. 2018.

[17] B. Libet, "Unconscious cerebral initiative and the role of conscious will in voluntary action," Behavioral and Brain Sciences, vol. 8, pp. 529-539, Dec. 1985. Publisher: Cambridge University Press. 